\title{
Intracranial haemorrhage in a 26 year-old woman with idiopathic thrombocytopenic purpura
}

\author{
Gavin Awerbuch and Reuven Sandyk
}

Department of Neurology, University of Arizona Health Sciences Center, Tucson, AZ. 85724, USA.

\begin{abstract}
Summary: Intracranial haemorrhage (ICH), a rare complication of idiopathic thrombocytopenic purpura (ITP), described only once previously in an adult, is usually fatal. We report a previously healthy 26 year old woman with chronic ITP in whom spontaneous ICH developed. The eventual favourable outcome in this case despite severe initial neurological deficit makes this case unusual. The importance of aggressive management in an ITP associated ICH is stressed and a plan for management is suggested.
\end{abstract}

\section{Introduction}

Although relatively rare, intracranial haemorrhage (ICH) is the most serious complication of idiopathic thrombocytopenic purpura (ITP) and is the leading reported cause of death. ${ }^{1-3}$ In children, nearly 20 cases of acute ITP complicated by ICH have been reported. Previous reports have alluded to ICH associated with ITP in adults, ${ }^{4-7}$ but ICH has been documented only once in adults. ${ }^{8}$ We report the occurrence of an intracerebral haemorrhage in a previous healthy adult with chronic ITP. The occurrence of ICH in ITP, the location of the haemorrhage and the favourable neurological outcome in this patient makes this case unusual. Further, a plan of management is suggested based on our experiences and review of previous publications.

\section{Case report}

A 26 year old black female presented with a 4-week history of bleeding gums, tarry stools, and easy bruising. No history of recent infections, myalgias, arthralgias, rash, fever, chills, photophobia or systemic lupus erythromatosus was obtained. She had not been taking any medications that could precipitate ITP. Four months prior to admission the patient delivered a normal male infant. There was no history of blood transfusion or thrombocytopenia at that time.

Examination revealed an acutely ill patient, who was afebrile with a blood pressure of $100 / 60 \mathrm{mmHg}$. There was diffuse purpura, ecchymosis and gum bleeding, but no rash. There were no retinal haemorrhages, splenomegaly or lymphadenopathy.

Laboratory investigations revealed a haemoglobin

Correspondence: Gavin Awerbuch M.D.

Accepted: 18 March 1987 of $5.7 \mathrm{~g} / \mathrm{dl}$, white blood count of $15.5 \times 10^{9} / 1$ with a normal differential and a platelet count of $6.0 \times 10^{9} / 1$, reticulocyte count was $3.2 \%$. The prothrombin time, partial thromboplastin time, thrombin time, fibrinogen, fibrin monomers, and fibrin split products were all normal. An electroencephalogram (EEG), gallium scan of the abdomen, chest X-ray, and computerized axial tomography (CT scan) of the head were normal. The bone marrow aspirate and biopsy showed increased megakaryocytes consistent with peripheral platelet destruction, but no other abnormality. Platelet antibody levels were not performed because of the profound thrombocytopenia. The work up for the aetiology of her thrombocytopenia including autoimmune and microbiological serology was completely negative and a diagnosis of acute ITP was made.

Considering the severity of the case and potential for a fatal outcome, therapy was initiated with 15 units of random donor platelets, 10 units of single donor platelets, and 2 units of packed red blood cell transfusions over the first 24 hours. Thrombocytopenia and mucosal bleeding continued and treatment was started with intravenous hydrocortisone, $150 \mathrm{mg}$ every 6 hours, intravenous gammaglobulin, $30 \mathrm{~g} / \mathrm{day}$, and a single dose of vincristine, $2 \mathrm{mg}$ intravenously. The patient did not respond to this therapy, attaining a maximum platelet count of $10 \times 10^{9} / 1$ during the first 6 days of hospitalization and was requiring up to 15 units of platelets daily. On hospital day 6, the patient developed a sudden onset of headache associated with confusion and disorientation. Neurological examination revealed normal fundi, and there were no focal neurological deficits. New subconjunctival haemorrhages were present. The platelet count was $2 \times 10^{9} / 1$. A clinical diagnosis of ICH was made. CT scan of the head showed bilateral basal ganglia haemorrhages 
(Figure 1). Treatment was started with intravenous dexamethasone $10 \mathrm{mg}$ every 6 hours and $20 \%$ mannitol $1 \mathrm{mg} / \mathrm{kg}$ to reduce cerebral oedema and intracranial pressure.

The patient was transfused with 20 units of platelets, two units of packed red blood cells and underwent emergency splenectomy. Post-operative treatment continued with dexamethasone, $10 \mathrm{mg}$ every 6 hours, platelet transfusions, 5-10 units every 24 hours, and intravenous gammaglobulin, $400 \mathrm{mg} / \mathrm{kg} / \mathrm{day}$. One day after surgery, the patient developed a right sided focal seizure involving the head and arm which lasted about 5 minutes. A second EEG showed a generalized dysrhythmia, delta grade II activity. A repeat CT scan showed no change. She was treated with phenytoin $300 \mathrm{mg} /$ day intravenously and no further seizures occurred. At this point, the patient was semi-comatose and over the next week remained in this state. However, during this period her platelet counts began to rise. The dexamethasone and gammaglobulin were slowly tapered over the next 2 weeks and eventually discontinued and no further bleeding episodes occurred. With improvement of her platelet counts, which rose to $75 \times 10^{9} / 1$, the neurological status improved. Three weeks post-operatively her platelet count reached $150 \times 10^{9} / 1$ and 6 weeks after admission $450 \times 10^{9} / 1$. Follow-up has continued for one year and the platelet counts and neurological examination have remained normal without any bleeding episodes.

\section{Discussion}

The most serious and life threatening complication of ITP is ICH ${ }^{1,9,13}$ Fortunately ICH is rare in ITP and probably occurs in less than $1 \%$ of all cases of ITP., ${ }^{2,3,10-13}$ It does however, account for $30-100 \%$ of fatalities in reported series. ${ }^{2,4,5,12}$ The haemorrhages are usually found in the subarachnoid areas and often they are multiple, varying in size from a single petechiae to large extravasations of blood. ${ }^{13}$ Posterior fossa haemorrhages are especially dangerous due to the possibility of rapid cerebellar herniation and brainstem compression. Retinal haemorrhages often occur at the time of the ICH. ${ }^{9}$

Factors predisposing to development of $\mathrm{ICH}$ are not well understood. Although trauma has accounted for some cases, most ICH seem to be spontaneous. ${ }^{2}$ ICH occurs most commonly in the early days after diagnosis, but can occur nearly anytime after onset. ${ }^{10,12}$ It has been suggested that patients with platelet counts of less than $10 \times 10^{9} / 1$ are at a greater risk of ICH. ${ }^{2,10,12,20}$

Previous authors have commented on the relative rarity and fatality of ICH in adults with ITP. For example, Difino et al. ${ }^{6}$ reported three incidences of ICH in his series of 67 adult cases of ITP, all of which

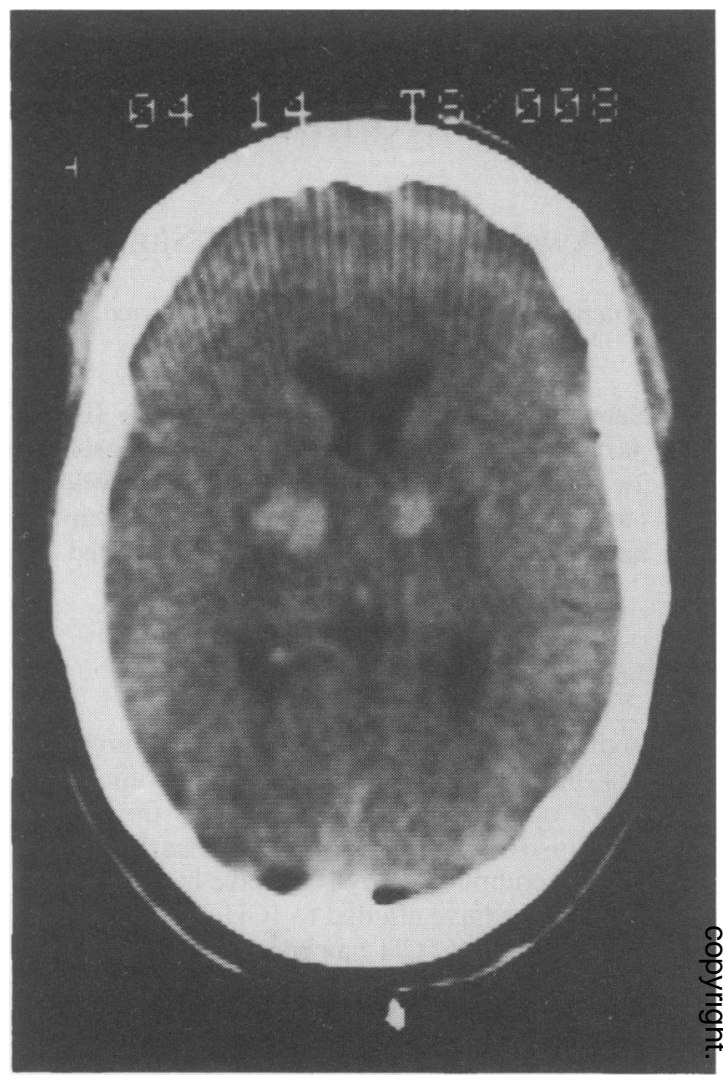

Figure 1 Unenhanced CT scan showing acute bilateral haemorrhages in the basal ganglia.

were fatal and Carpenter et al. ${ }^{5}$ reported two cases of ICH in his series of 85 adults and both were fatal. However, they gave no specific details as to how the diagnosis of the ICH was made, the clinical course, or the treatment. Other reports also allude to adult cases of ITP complicated by ICH, but this diagnosis was uncertain because no investigational tests (e.g. CT scan) were performed to prove that haemorrhages had occurred..$^{4-7}$ Further, with the current knowledge of the disease, it is questionable whether some of the older reports of ICH were truly examples of ITP or whether they represented other diseases which may have presented as thrombocytopenia. More recently, Nagler $e t$ al. ${ }^{8}$ reported the occurrence of a subdural haematoma in a 32 year old man with chronic ITP. This is the only other documented case of an ICH occurring in an adult with ITP.

If a patient with ITP develops signs or symptoms of central nervous system bleeding such as retinal haemorrhages, convulsions, meningismus, headache, personality changes or neurological signs, rapid and vigorous treatment must be initiated as this can be 
fatal. Therapy for ICH associated with ITP consists of controlling the elevated intracranial pressure while also achieving a rapid rise in the platelet count to control the bleeding. ${ }^{15}$ Traditional methods of head elevation, fluid restrictions, avoidance of hypercapnia and intravenous dexamethasone and $20 \%$ mannitol can be implemented to control cerebral oedema. ${ }^{14}$ Platelet transfusion alone may slow down the bleeding but it seldom results in a sustained rise of platelet count due to peripheral platelet destruction..$^{12,16,19}$ Steroid and immunosupressive therapy appear to increase platelet life span but no controlled studies show that this reduces the risk of ICH. ${ }^{2,16-18}$ Emergency splenectomy followed by platelet concentrates is employed in cases of ITP refractory to conservative therapy, or if life threatening haemorrhages occur., ${ }^{2,14,19}$ The response of the platelet count to splenectomy varies from $70-90 \%,{ }^{19,20}$ and is usually immediate with peak counts obtained in 1-2 weeks. ${ }^{12,19}$ If the level of consciousness deteriorates, or neurological deficits

\section{References}

1. Komrower, G.M. \& Watson, G.H. Prognosis in idiopathic thrombocytopenic purpura of childhood. Arch Dis Child 1954, 29: 502-506.

2. Woerner, S.J., Abildguard, C.F. \& French, B.N. Intracranial haemorrhage in children with idiopathic thrombocytopenic purpura. Pediatrics 1981, 67: 453460.

3. Lusher, J.M. \& Zuelzer, W.W. Idiopathic thrombocytopenic purpura in childhood. $J$ Pediatr 1966, 68: 971-979.

4. Doan, C.A., $\quad$ Bouroncle, B.A. \& Wiseman, B.K. Idiopathic and secondary thrombocytopenic purpura: Clinical study and evaluation of 381 cases over a period of 28 years. Ann Intern Med 1960, 53: 861-876.

5. Carpenter, A.F., Wintrobe, M.M., Fuller, E.A., Hart, A. \& Cartwright, G.E. Treatment of idiopathic thrombocytopenic purpura. JAMA 1959, 171: 1911-1916.

6. Difino, S.M., Lachant, N.A., Kirshner, J.J. \& Gootleib, A.J. Adult idiopathic thrombocytopenic purpura: clinical findings and response to therapy. Am J Med 1980, 69: 430-442.

7. Hirsh, E.O. \& Dameshek, W. Idiopathic thrombocytopenia. Review of 89 cases with particular reference to the differentiation and treatment of acute (self limited) and chronic types. Arch Int Med 1951, 88: $701-728$.

8. Nagler, A., Brenner, B., Hayek, T. \& Brook, J.G. Subdural hematoma in a 32 year old man with chronic idiopathic thrombocytopenic purpura. Acta Haematol (Basel) 1986, 74: 186-187.

9. Mueller-Eckhardt, C. Idiopathic thrombocytopenic purpura: clinical and immunologic considerations. Semin Thromb Hemost 1977, 3: 125-159.

10. Lusher, L.M. \& Iyer, R. Idiopathic thrombocytopenic purpura in children. Semin Thromb Hemost 1977, 3: 175194. occur and the haemorrhage is in an accessible location, neurosurgical intervention may be warranted. Haemorrhages into the posterior fossa should usually be treated with craniotomy due to the possibility of a rapid rise in intracranial pressure. ${ }^{2}$ It has been stressed that splenectomy should be done prior to craniotomy to avoid further ICH during surgery although both procedures may be done together under one anaesthesia. ${ }^{14,20}$

As ICH is the most dreaded complication of ITP, we thought it valuable to examine our experience in this case with a successful outcome. Our case demonstrates that successful treatment of the ICH depends on early diagnosis and prompt, aggressive management of the bleeding. If central nervous system symptoms are present, the alert physician with use of the present diagnostic techniques, and medical and surgical management, may be able to prevent permanent neurological deficit in an effective manner.

11. Dameshk, W. Controversies in idiopathic thrombocytopenic purpura. JAMA 1960, 173: 1025-1029.

12. McClure, P.D. Idiopathic thrombocytopenic purpura in childhood: diagnosis and management. Pediatrics 1975, 55: $68-74$

13. Wintrobe, $M$. Idiopathic thrombocytopenic purpura. In Wintrobe, M. (ed) Clinical Hematology, 7th edition. Lea \& Febiger, Philadelphia, 1974, p 1075.

14. Humphreys, R.P., Hockley, A.D., Freedman, M.H. \& Saunders, E.F. Management of intracranial haemorrhage in idiopathic thrombocytopenic purpura. $J$ Neurosurg 1976, 45: 700-704.

15. Lotan, C.S., Wald, U., Ludomirsky, A. \& Cividalli, G. Intracranial hemorrhage simulating brain tumor in immune thrombocytopenic purpura. Eur J Pediatr 1983, 141: $127-128$.

16. Dunn, N.L. \& Maurer, H.M. Prednisone treatment of acute idiopathic thrombocytopenic purpura in childhood. Advantages. Am J Pediat Hematol Oncol 1984, 6: 159-164.

17. Oral, A., Nusbacher, J., Hill, J.B. \& Lewis, J.H. Intravenous gammaglobulin in the treatment of chronic idiopathic thrombocytopenic purpura in adults. Am J Med 1984, 30: 187-192.

18. Mchino, H., Yasunaga, K. \& Akatsuka, J.A. A cooperative clinical trial of high dose immunoglobulin therapy in 177 cases of idiopathic thrombocytopenic purpura. Thromb Haemost 1984, 51: 182-519.

19. Lacey, J.V. \& Penner, J.A. Management of idiopathic thrombocytopenic purpura in the adult. Semin Thromb Hemost 1977, 3: 160-174.

20. McMillan, R. Chronic idiopathic thrombocytopenic purpura. $N$ Engl J Med 1981, 304: 1135-1147. 